\title{
Role of Oral Microbiome in Disease Predictions - Current Advances
}

\author{
Kavita Pandey ${ }^{1}$, Rajesh Pandey ${ }^{1}$, Subhash Padhye ${ }^{2,3 *}$, Shrikant Anant ${ }^{3}$ and Shahid Umar ${ }^{4}$ \\ ${ }^{1}$ Dept of Bioanalytical Sciences (Integrated), Guru Nanak Khalsa College, Matunga, Mumbai, India \\ ${ }^{2}$ Interdisciplinary Science \& Technology Research Academy, India
}

${ }^{3}$ Department of Cancer biology, University of Kansas Medical Center, Kansas, USA

${ }^{4}$ Department of Surgery, University of Kansas Medical Center, Kansas, USA

*Corresponding author: Subhash Padhye, Interdisciplinary Science \& Technology Research Academy, India

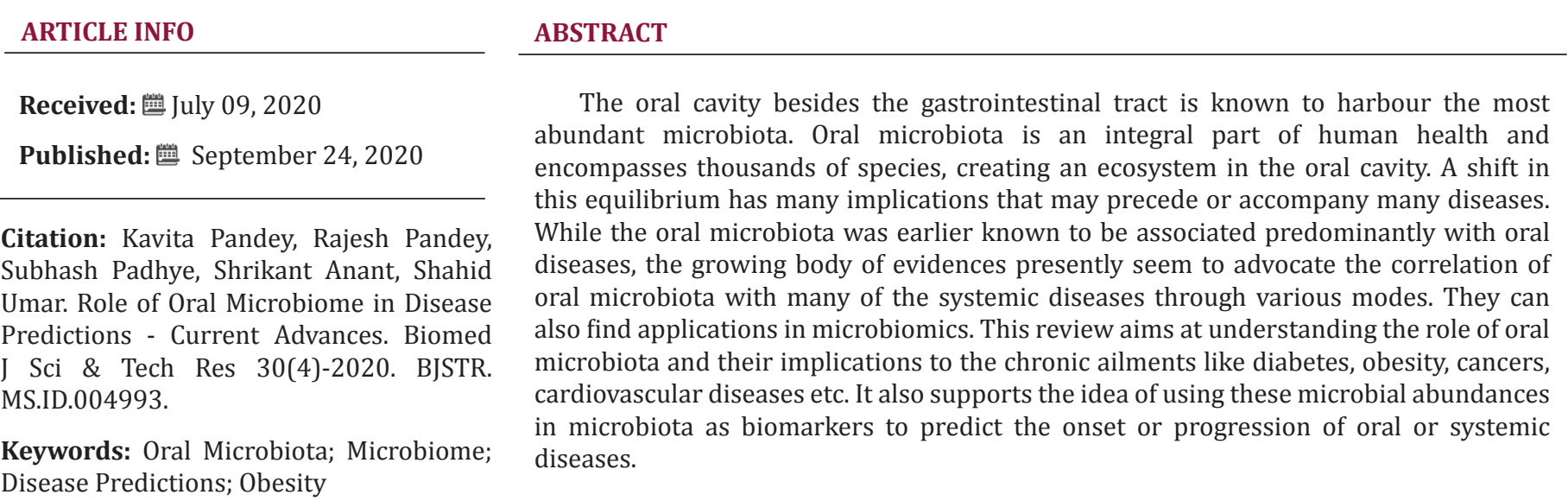

\section{Introduction}

The human body is more of microbes and less of humans. Over the millions of years, microbiome and the human body have coevolved for the betterment of each other. Human gastrointestinal tract (GIT) harbours the most abundant number and varieties of microbes. The second most abundance is found in the oral cavity. The oral microbiota is dynamic and comprises of spectrum of microbial species possessing functions ranging from acidogenic, acidouric, inflammatory, and anti-inflammatory activities. A sum of all the microbes present in oral cavity (habitats like buccal mucosa, hard palate, gingiva, tonsils, saliva, sub-lingual, throat and tongue dorsum) is what comprises the oral microbiota and their genomes collectively constitutes the oral microbiome. Today, the oral microbiome is an expanding field of research based on the pioneering work of Dutchman Antony van Leeuwenhoek. He was the first to study the microbes in his tooth plaque using his selfconstructed microscope. Reports that he submitted to the Royal society, in 1670s, revealed that several forms of microorganisms were present on the tooth surface[1].

The human oral flora comprises of hundreds of microbial species ranging from bacteria, archaea, fungi, protozoa to viruses. Table 1 enlists some of the more dominating phyla of the oral microbiota. The technological properties of the oral microbiota have been recently reviwed by Arweiler and Netuschil, [2].A balanced ecosystem exists among the microorganisms in oral cavity, which is responsible for health. However, destruction of this equilibrium (dysbiosis) encourages growth of pathogens. The composition of oral microbiota keeps evolving throughout life, but the early oral microbiome dictates the composition of longterm stable adult oral microbiome[3,4]. Perhaps, the first dynamic alteration of oral microbiota was due to shifts in diet, as is the case with increased intake of sugars, consumption of processed or junk foods etc[5]. Several other factors influence the composition 
of the oral microbiota, including diet, oral hygiene, smoking, age, use of antimicrobials and vaccines, construction of biomaterials like denture, implants etc.[6]. Host genetics may also influence this composition [7] along with availability of nutrients, oxygen tension, host immunological factors etc. Oral microbiota composition reflects the host's immune status and diversity and perturbations

Table 1: Major genera of bacterial species present in oral microbiota. in the abundance of specific strains of the oral microbiota, can serve as 'biomarkers' that can be used to predict several diseases. The rapidly growing area of application of microbiome in therapeutic applications is called microbiomics, where oral microbiota holds a great potential.

\begin{tabular}{|c|c|c|c|c|}
\hline $\begin{array}{c}\text { Gram nature } \rightarrow \\
\text { Shape } \downarrow\end{array}$ & \multicolumn{2}{|c|}{ Gram positive } & Moraxella, \\
Cocci & Abiotrophia, & Streptococcus, \\
Peptostreptococcus, & Stomatococcus & Neisseria, & Veillonella \\
\hline & & & Campylobacter, & Hemophilus, \\
& Actinomyces, & Lactobacillus, & Capnocytophaga, & Prevotella, \\
& Bifidobacterium, & Propionibacterium, & Desulfobacter, & Selemonas, \\
& Corynebacterium, & Pseudoramibacter, & Desulfovibrio, & Simonsiella, \\
& Eubacterium, & Rothia & Eikenella, & Fusobacterium, \\
& & & & Treponema, \\
& & & Wolinella. \\
\hline
\end{tabular}

Early diagnosis and prompt treatment of several chronic diseases are essential to prevent or delay complications by making timely lifestyle changes and delaying or possibly preventing these diseases like diabetes, hypertension or obesity [8,9].Oral cavity including soft and hard tissues shows early and delayed signs and symptoms of these ailments; however, awareness of these complications is lacking worldwide and needs further scale of understanding. In depth understanding of the disease development and involved pathways coupled with pharmacological response to therapeutic interventions has enabled the scientific community to identify biomarkers that holds great promise in predictive medicines[10-12]. Advent of omics technologies (metagenomics, transcriptomics, metabolomics etc) has made it even easier to identify and measure such biomarkers[13,14].

Some of the prominent representatives of the oral microbiota include the phyla Actinobacteria (Atopobium, Cryptobacterium, Kocuria, Olsenella, Parascardovia, Scardovia, Slackia, Tropheryma),Firmicutes (Abiotrophia, Anaerococcus, Aneroglobus, Bulleidia, Catonella, Dialister, Filifactor, Finegoldia, Granulicatella, Johnsonella, Mogibacterium, Parvimonas, Peptoniphilus, Pseudoramibacter, Schwartzia,Shuttleworthia, Solobacterium), Bacteroidetes (Bergeyella, Prevotella, Tannerella), Proteobacteria(Lautropia, Suttonella) and Synergistetes(Jonquetella, Pyramidobacter) (Adapted from Arweiler and Netuschil, (2016)Comprehending the importance of human microbiome, the National Institute of Health (NIH) in 2008, launched the Human Microbiome Project (HMP).Expanded Human Oral Microbiome Database (eHOMD), is the updated database with comprehensive curated information on the microbial species present in the human aerodigestive tract (ADT). It encompasses a total of 771 microbial species, of all the species, $57 \%$ are officially named, $13 \%$ unnamed but cultivated and $30 \%$ are known only as uncultivated phylotypes [15-17]Most abundant bacterial genera present in a healthy oral cavity are enlisted in table 1 . It is this complex and intricate relationship between the host and the oral microbiota that decides the health status of an individual [18]. Multiple omics technologies have been adopted, data collected has been integrated and analysed to achiever deeper understanding of the microbiota and its interactions with the hosts [19].

\section{Obesity}

Abnormal or excessive accumulation of fats leading to increased risks of many other severe ailments is termed as obesity. Obesity is a global healthcare pandemic and is no more limited to high income countries alone. It is also one of the primary risk factors to an array of chronic conditions like periodontics, cardiovascular diseases, cancer, diabetes, rheumatoid arthritis etc. Adipose tissue, earlier thought to be only storing fats, has now been discovered to be a complex and metabolically active endocrine gland, which secretes several immuno-modulatory factors[20]. A strong causal link exists between excess adiposity and several metabolic diseases as depicted in (Figure 1) [21]. Adipose and related cells secrete over 50 bioactive molecules termed as adipokines, the concentration of which partly defines the individual's metabolic health[22,23]. Low-grade inflammation is a guaranteed characteristic of adult obesity.Alterations in the oral microbiome have potential in sentinel diagnostic and prognostic applications, especially in case of predicting obesity [24-26]. 


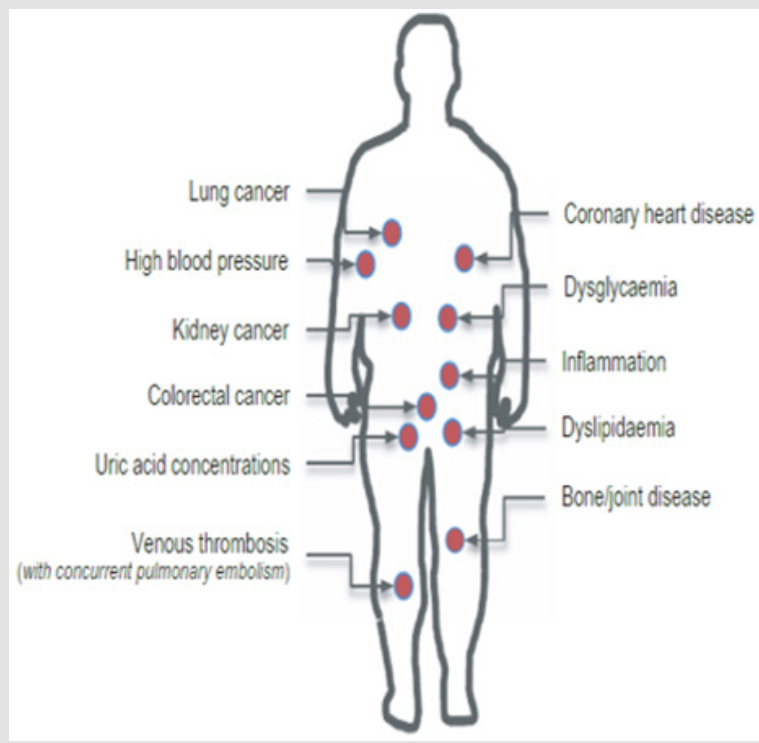

Figure 1: Causal link between excessive adiposity in health.

Literature cites several studies that have associated obesity with dysbiosis in the oral (and gut microbiomes). Oral cavity in obese individuals has higher levels of several bacteria than in nonobese controls, and it seems likely that those bacterial species could serve as biological indicators of obesity. Obesity may be a factor contributing to periodontitis severity via a modulation of the immune system and vice versa [27].The family Gemellaceae has been reported to be more abundant in saliva samples from obese participants than in normal weight individuals[28].Many studies have concluded that oral microbiome composition significantly differed between obese and non-obese patients. Dominance of Bacteroidetes, Spirochaetes and Firmicutes in the subgingival plaque acts as a biomarker for identification or early detection of obesity. Amongst the oral microflora, over representation of Proteobacteria, Chloroflexi and Firmicutes, with the noted absence of representatives from the phylum Bacteroidetes also reflects risks of obesity. Notably, the phylum Firmicutes was identified in obese patients as an independent significantly discriminative feature with an abundance of over four orders of magnitude [29]. In saliva, [30] identified Selenomonasnoxia, a representative of the Firmicutes phylum, to be a robust predictor of obesity.

In another recent study by Sohail and team (2019), one of the striking observations made was the higher Firmicutes/ Bacteroidetes ratio, in the obese subjects. Tam and co-workers, reported the abundance of Firmicutes phylum (as high as 4 orders) in obese subjects, thus was the discriminative factor and can serve as a biomarker[31]. Chemerin levels in Gingival crevicular fluid (GCF), is indicative of glucose, lipid metabolism and inflammation status of an individual [32]. It is responsible for adipocyte differentiation and adipogenesis. Chemerin supports the connection of macrophages to endothelia tissues. Obese people are known to display fluctuating levels of chemerin in multiple studies (???).Maternal obesity is related to immunologic and inflammatory systemic modifications that may worsen the pregnancy inflammatory status [33]. Polycystic ovary syndrome (PCOS) is a lifestyle disorder affecting 1 in 5 women, often complicated by infertility, diabetes and immunological issues but very commonlyobesity.

\section{Diabetes}

It is predicted that by next decade (2030), diabetes may affect 79.4 million individuals in India alone. A bidirectional relationship exists between periodontal disease(s) and diabetes [34]. Studies undertaken to investigate the implications of oral microbiota on diabetes development are scanty and need more attention [35]. In a latest randomised controlled trial,de Groot and team (2020) concluded that the oral supplementation of butyrate had significant effect on the faecal butyrate and propionate levels. Noteworthy changes in subgingival microbiota between type 2 diabetes mellitus (T2DM) subjects and non-diabetic controls was reported by Casarin and team. The species included-TM7, Actinomyces, Aggregatibacter, Capnocytophaga, Eikenella, Fusobacterium, Gemella, Neisseria, Selenomonas, Streptococcus and Veillonella genera [36,37].

Higher abundances of the genus Granulicatella, in the family Gemellaceae, was observed in diabetic and non-diabetic obese participants compared to the normal weight controls [38,39]. Inverse relationship exists between abundance of the genus Bifidobacteria in the phylum Actinobacteria and diabetes $[40,50]$. Another study by Kampoo and coworkers reported significantly higher abundances of two genera in the phylum FirmicutesStreptococcus and Lactobacillus in diabetic patients, indicating their use as markers to predict diabetes. Multiple studies have correlated the dominance of periodontal pathogenic bacteria -P. gingivalis, Treponema denticola and Tannerellaforsythiawith diabetes risk and glycemic control [51-55]. In general, higher abundances of most taxa in the phylum Actinobacteria were associated with a decreased risk of diabetes [56].

\section{Oral Cancer}

Squamous cell carcinoma is the most frequently occurring malignancy of the oral cavity and adjacent sites, representing over $90 \%$ of all cancers [57]. Several studies, have correlated prevalence of certain species of oral microbiota species (likeAggregatibacter, Actinobacillus, Actinomyces, Capnocytophaga, Fusobacterium, Haemophilus, Leptotrichia, Oribacterium, Neisseria, Porphyromonas, Rothia and Veillonella), in patients with head and neck cancers (HNC) [58-62].Mager and coworkers suggested that high salivary counts of Prevotellamelaninogenica, Streptococcus mitis and Capnocytophagagingivaliscould act as diagnostic indicators of oral squamous cell carcinoma (OSCC). Another work by Gholizadeh and team confirmed the link between prevalence 
of oral pathogens like Porphyromonasgingivalis, Fusobacterium nucleatum, Prevotella intermedia and OSCC $[63,64]$.

Several other types of cancers are also associated with alterations in oral microbiota. [65], in a recent study, reported that the oral microbiome panel of Capnocytophaga, Haemophilus, Corynebacterium, Rothia, Porphyromonas, Paludibacter and Oribacterium, acted as biomarkers by being able to very accurately, discriminate age-matched normal healthy controls from patients suffering from oral cavity cancer (OCC) and oropharyngial cancers (OPC). Additionally, loss of abundance of Paludibacter and Corynebacterium was also reported for the first time.A study conducted by Yan et al.concluded that significantly higher levels of oral pathogens Capnocytophaga and Veillonella in salivary microbiome were associated with non-small cell lung cancer (NSCLC). Periodontal pathogens- Porphyromonasgingivalis and Tannerella forsythia have been associated with increased risk of esophageal adenocarcinoma (EAC) and esophageal squamous cellcarcinomas (ESCC), while decreased counts of strains of genus Neisseria and Streptococcus pneumoniae were associated with lowered risk of EAC and ESCC development [66].

Significantly higher counts of oral pathogens Porphyromonasgingivalis and Aggregatibacteractinomycetemcomitans have been associated with pancreatic cancer (Ref?). Lower counts of Leptotrichia genus on the other hand, was associated with decreased risk of pancreatic cancer $[67,68]$. compared the microbiota in saliva from healthy controls and pancreatic cancer patients, where they identified 2 bacteria (Streptococcus mitis and Neisseria elongata) in combination which could act as biomarkers because of their ability to distinguish between pancreatic cancer cases and control group.

\section{Gastrointestinal Diseases}

More and more diseases of the gastrointestinal tract are associated with oral microbiome. Inflammatory bowel disease (IBD) is one of the first and most studied one. IBD patients are very commonly observed to suffer from oral symptoms like ulcers or aphthous stomatitis. Also strains like Pyostomatitisvegetans are found in high abundances [69], however it needs more data to confirm. Dominance of salivary microbiota with genera Bacteroidetes, Gemella, Haemophilus, Neisseria Prevotella, Streptococcus and Veillonella, were found to cause dysbiosis observed in IBD patients[70]. Gastrointestinal cancer risk increases in individuals with periodontal disease or tooth loss, conditions caused by oral bacteria indicating the role of oral microbiota in development of gastrointestinal ailments. Saliva microbiome shows higher abundance of species of family Gemellaceae in patients suffering from Crohn's disease [71].Colorectal cancer (CRC) and gastric cancer (GC), the two most common types of GI cancer, accounted for about 2.8million new cases and 1.6 million deaths worldwide, respectively, in 2018 [73] and has been correlated with fluctuations in oral microbiota.

\section{Cardiovascular Diseases}

Atherosclerotic diseases like stroke and myocardial infarction are amongst the main causes of death worldwide. Several studies on the these aspects have collectively detected the following species associated with cardiovascular diseasesAggregatibacteractinomycetemcomitans,

Fusobacterium nucleatum, Porphyromonasgingivalis, Prevotella intermedia, and Tannerella forsythia. In particular, predominance of A actinomycetemcomitans (facultative aerobe) and $\mathrm{P}$ gingivalis, (obligate anaerobe), has been associated with atherosclerosis. Abundance of Anaeroglobus has been correlated to symptomatic atherosclerosis[73].In certain studies infectious agents like Chlamydophyla pneumonia, Treponema denticoland Cytomegalovirus also have been implicated with cardiovascular problems In such infections, prolonged periodontal treatments influencing the oral hygiene habits lead to reduction in oral anaerobes, and decreased inflammatory biomarkers. In some cases, thickening of the carotid artery (associated with stroke) was reversed.

\section{Adverse Pregnancy Outcomes (Apos) And Post-Partum}

Several Adverse pregnancy outcomes (APOs) have been correlated withfluctuations in oral microbiota of pregnant females. The increased counts of Bacteroides forsythusand Campylobacter rectus elicit systemic inflammatory responses in pregnant females at the foeto-placental junction, thereby increasing the risk of APOs in pregnant women. Another study by Han and co-workers revealed that F. nucleatum (an oral pathogen), escaped into blood of pregnant women and reached the placenta, resulting in stillbirths. Gestational diabetes mellitus (GDM) has been associated with minor aberrations of the salivary microbiota during third trimester and postpartum, which is correlated to the physiological changes of the immune system during pregnancy.

Microbiome of the breast milk (having many good bacteria, varies throughout lactation and is known to shape the oral microbiota of the individual. Ofcourse environmental factors have their own influences too. The saliva microbiome differs between breast-fed and formula-fed 3-month-old infantsand breast-fed children were found to be less affected by dental caries. Study by $\mathrm{Xu}$ and team (2019), advocate the presence of non-invasive biomarkers in the oral (and intestinal) microbiome, which aid in monitoring health management of Gestational diabetes mellitus in pregnant women.

\section{Periodontal Diseases}

Periodontal diseases are pathological inflammatory conditions of the periodontal tissues (gum and bone support surrounding the teeth). Gingivitis (inflammation of the gum at the necks of the teeth) and periodontitis (inflammation affecting the bone and tissues of the teeth) are two of the most prevalent periodontal diseases. In response to the infection by specific oral pathogens and their toxins, 
the individual's immune- inflammatory system gets stimulated. The lipopolysaccharides layer (LPS) of Gram negative pathogens triggers the production of several pro-inflammatory mediators resulting in a local effect, destruction of extracellular matrix and alveolar bone. These molecules have been proven to further exert systemic effects like increased risks of atherosclerosis and insulin resistance. Thus the periodontal disease may commence as a local infection but by triggering the chronic inflammatory responses it may end up in blood stream, eventually making the host more susceptible to acquire conditions like cardiovascular diseases, diabetes, obesity, rheumatoid arthritis, arterial diseases, osteoporosis, mental disorders, cerebrovascular diseases and complications in pregnancy. Though scanty, but emerging evidences support the correlation between periodontitis and systemic diseases. The genus Gemella has recently been reported to be among the genera most predictive of dysbiosis[62].

\section{Bacteraemia}

Presence of bacteria in blood is termed bacteraemia. Correlation between oral dysbiosis and periodontitis is a well-established fact. Oral pathogens (like several Streptococcus species and the Gram negative rods H. parainfluenzae, Aggregatibacteraphrophilus and A. actinomycetemcomitans), after escaping into the blood stream in some cases have been found to settle on formerly damaged heart valves and result in infective endocarditis (Gao et al., 2018), or form thrombi and pustules in the brain. Peri-implantitis (infectious causing inflammation of the tissues surrounding the implant) is also a result of abundance of some species in the oral microbiotaEubacteriumminutum, Prevotella intermedia. Atherosclerotic plaques have been found to be colonized by bacteria associated with periodontitis, suggesting correlation of oral microbiota with cardiovascular diseases. Septicaemia in Leukaemia patients also has possible correlation with the oral flora.

\section{Brain Health}

[51], studied the oral and skin microbiota of aging males and females. Many microbial markers(such as ASVs belonging to Lactobacillales, Gemellaceae, Bacteroides, and Fusobacterium), declined with age. Population displaying healthy aging are found to have higher $\alpha$-diversity compared to non-healthy aging.Literature cites several reports, advocating the correlation between oral health of an individual to mental health, especially in aging population. The mouth, through teeth, connects the oral environment directly to bones. As we age, our humoral and cell mediated immune systems become weaker and gradually only innate immunity predominates. This also affects the way one ages. Oral dysbiosis has been correlated to cognitive decline in many studies especially in patients with Alzheimer's disease (AD), associated with neuroinflammation presumptively caused by the release of inflammatory mediators by oral microbiota. One of the mechanisms states that the oral bacteria or their endotoxins gain entry to the brain, resulting in microglial activation (especially astrocytes). This, in turn triggers the production of pro-inflammatory cytokines such as TNF $\alpha$ and IL1 and apolipoprotein E (ApoE). Prolonged exposure to high concentrations of $\mathrm{TNF} \alpha$ weakens the protective blood-brainbarrier (BBB) making it even more prone to entrance of bacteria or endotoxins. This continuous cascade of events, over the months or years, results in neuronal damage.

Increased abundance of Treponema and presence of lipopolysaccharides from oral anaerobe Porphyromonasgingivalis has been consistently observed in AD patients. Current diagnostic methods to identify AD include - detecting Volatileorganic compounds (VOCs)secreted in each breath, salivary lipidomics, salivary proteins like A $\beta$ peptides, salivary acetyl cholinesterase etc and most importantly the composition of oral microbiome.

\section{Conclusions}

Research in the area of oral microbiome has led to paradigm shift in attention from oral to systemic diseases. The time demands development of multifactorial methods of re-establishing the harmonious community in the oral cavity. Some of the ways include achieving good oral hygiene, changes in diet such that sucrose is minimum, quitting addictions like smoking and alcoholism, boosting our immune system etc. A growing body of evidence suggests that alterations in the oral microbiome have potential in accurate diagnostic and prognostic applications. The molecular biology and sequencing technologies have expedited the pace of exploration of oral microbiome. Data generated needs to be effectively utilized for developing microbiome-based biomarkers for targeted patient management and more importantly to be used in disease prediction. While this opens up the arena of personalized medicines, a deeper understanding of the cross talks between host and the oral microbiome may help facilitate development of oral microbiome transplant to manage oral diseases.

\section{References}

1. Fåk F, Tremaroli V, Bergström G, Bäckhed F (2015) Oral microbiota in patients with atherosclerosis. Atherosclerosis 243(2): 573-578.

2. Alcaraz LD, Belda-Ferre P, Cabrera-Rubio R, Romero H, Simón-Soro Á, et al. (2012) Identifying a healthy oral microbiome through metagenomics. Clinical Microbiology and Infection 18(4): 54-57.

3. Arweiler NB, Netuschil L (2016) The oral microbiota. In Microbiota of the Human Body pp: 45-60.

4. Avila WM, Pordeus IA, Paiva SM, Martins CC (2015) Breast and bottle feeding as risk factors for dental caries: a systematic review and metaanalysis. PloS one 10(11): e0142922.

5. Balmasova IP, Lomakin YA, Babaev EA, Tsarev VN, Gabibov AG, et al. (2019) "Shielding" of Cytokine Induction by the Periodontal Microbiome in Patients with Periodontitis Associated with Type 2 Diabetes Mellitus. Acta Naturae 11(4): 79-87.

6. Bik EM, Long CD, Armitage GC, Loomer P, Emerson J, (2010) Bacterial diversity in the oral cavity of 10 healthy individuals. The ISME journal 4(8): 962-974.

7. Casarin RCV, Barbagallo A, Meulman T, Santos VR, Sallum EA, (2013) Subgingival biodiversity in subjects with uncontrolled type-2 diabetes and chronic periodontitis. Journal of periodontal research 48(1): 30-36. 
8. Chen Y, Chen X, Yu H, Zhou H, Xu S, et al. (2019) Oral Microbiota as Promising Diagnostic Biomarkers for Gastrointestinal Cancer: A Systematic Review. OncoTargets and therapy 12: 11131-11144.

9. Schara R, Skaleric E, Seme K, Skaleric U (2013) Prevalence of periodontal pathogens and metabolic control of type 1 diabetes patients. Journal of the International Academy of Periodontology 15(1): 29-34.

10. Hyvärinen K, Salminen A, Salomaa V, Pussinen PJ (2015) Systemic exposure to a common periodontal pathogen and missing teeth are associated with metabolic syndrome. Acta diabetologica 52(1): 179-182.

11. Makiura N, Ojima M, Kou Y, Furuta N, Okahashi N, (2008) Relationship of Porphyromonasgingivalis with glycemic level in patients with type 2 diabetes following periodontal treatment. Oral microbiology and immunology 23(4): 348-351.

12. Aemaimanan P, Amimanan P, Taweechaisupapong S (2013) Quantification of key periodontal pathogens in insulin-dependent type 2 diabetic and non-diabetic patients with generalized chronic periodontitis. Anaerobe 22: 64-68.

13. Kampoo K, Teanpaisan R, Ledder RG, McBain AJ (2014) Oral bacterial communities in individuals with type 2 diabetes who live in southern Thailand. Appl Environ Microbiol 80(2): 662-671.

14. Shillitoe E, Weinstock R, Kim T, Simon H, Planer J (2012) The ora microflora in obesity and type-2 diabetes. Journal of oral microbiology 4(1): 19013.

15. Long, J, Cai Q Steinwandel M, Hargreaves MK, Bordenstein SR, et al. (2017) Association of oral microbiome with type 2 diabetes risk. Journal of periodontal research 52(3): 636-643.

16. Piombino P, Genovese A, Esposito S, Moio L, Cutolo PP, et al. (2014) Saliva from obese individuals suppresses the release of aroma compounds from wine. PloS one $9(1)$

17. Haberman Y, Tickle TL, Dexheimer PJ, Kim M O, Tang D, et al. (2014) Pediatric Crohn disease patients exhibit specific ileal transcriptome and microbiome signature. The Journal of clinical investigation 124(8): 3617-3633.

18. Crusell MK, Brink LR, Nielsen T, Allin KH, Hansen T, et al. (2020) Gestational diabetes and the human salivary microbiota: a longitudinal study during pregnancy and postpartum. BMC Pregnancy and Childbirth, 20(1): 69.

19. Davé V, Street K, Francis S, Bradman A, Riley L, et al. (2016) Bacterial microbiome of breast milk and child saliva from low-income Mexican American women and children. Pediatric research 79(6): 846-854.

20. De Groot PF, Nikolic T, Imangaliyev S, Bekkering S, Duinkerken G, et al. (2020) Oral butyrate does not affect innate immunity and islet autoimmunity in individuals with longstanding type 1 diabetes: a randomised controlled trial. Diabetologia 63(3): 597-610.

21. Dickstein JB, Moldofsky H, Hay JB (2000) Brain-blood permeability: TNF- $\alpha$ promotes escape of protein tracer from CSF to blood. American Journal of Physiology-Regulatory, Integrative and Comparative Physiology 279(1): R148-R151.

22. Fan X, Alekseyenko AV, Wu J, Peters BA, Jacobs EJ, et al. (2018) Human oral microbiome and prospective risk for pancreatic cancer: a populationbased nested case-control study. Gut 67(1): 120-127.

23. Galvão-Moreira LV, da Cruz MC FN (2016) Oral microbiome, periodontitis and risk of head and neck cancer. Oral oncology 53: 17-19.

24. Furquim CP, Soares GMS, Ribeiro LL, Azcarate-Peril MA, Butz N, et al. (2017) The salivary microbiome and oral cancer risk: A pilot study in fanconianemia. Journal of dental research 96(3): 292-299.

25. Giannobile WV (2012) Salivary diagnostics for periodontal diseases. The Journal of the American Dental Association 143: 6S-11S.

26. Gomes AS, Sampaio-Maia B, Vasconcelos M, Fonesca PA, Figueiral H, et al. (2015) In situ evaluation of the microbial adhesion on a hard acrylic resin and a soft liner used in removable prostheses. Int J Prosthodont, 28(1): 65-71.
27. Griffen AL, Beall CJ, Campbell JH, Firestone ND, Kumar PS, et al. (2012) Distinct and complex bacterial profiles in human periodontitis and health revealed by $16 \mathrm{~S}$ pyrosequencing. The ISME journal 6(6): 11761185 .

28. Guerrero-Preston R, Sidransky D (2017) U.S. Patent Application No. $15 / 128,439$.

29. Hall MW, Singh N, Ng KF, Lam DK, Goldberg MB, et al. (2017) Interpersonal diversity and temporal dynamics of dental, tongue, and salivary microbiota in the healthy oral cavity. NPJ biofilms and microbiomes 3(1): 1-7.

30. Han YW, Fardini Y, Chen C, Iacampo KG, Peraino VA, et al. (2010) Term stillbirth caused by oral Fusobacterium nucleatum. Obstetrics and gynecology 115(2 Pt 2): 442-445.

31. He Z, Wu J, Xiao B, Xiao S, Li H, Wu K (2019) The Initial Oral Microbiota of Neonates Among Subjects With Gestational Diabetes Mellitus. Frontiers in Pediatrics 7: 513.

32. Iaffaldano L, Granata I, Pagliuca C, Esposito MV, Casaburi G, et al. (2018) Oropharyngeal microbiome evaluation highlights Neisseria abundance in active celiac patients. Scientific reports 8(1): 1-10.

33. Jeurink PV, Van Bergenhenegouwen J, Jiménez E, Knippels LMJ, Fernández L, et al. (2013) Human milk: a source of more life than we imagine. Beneficial microbes 4(1): 17-30.

34. Joshipura KJ, Wand HC, Merchant AT, Rimm EB (2004) Periodontal disease and biomarkers related to cardiovascular disease. Journal of dental research 83(2): 151-155.

35. Kilian M (2018) The oral microbiome-friend or foe? European journal of oral sciences 126: 5-12.

36. Kilian M, Chapple IL C, Hannig M, Marsh PD, Meuric V, et al. (2016) The oral microbiome-an update for oral healthcare professionals. British dental journal 221(10): 657-666.

37. Kodukula K, Faller DV, Harpp DN, Kanara I, Pernokas J, Pernokas M, et al. (2017) Gut microbiota and salivary diagnostics: the mouth is salivating to tell us something. BioResearch open access 6(1). 123-132.

38. Krishnan K, Chen T, Paster BJ (2017) A practical guide to the oral microbiome and its relation to health and disease. Oral diseases 23(3): 276-286.

39. Kumar PS, Mason MR (2015) Mouthguards: does the indigenous microbiome play a role in maintaining oral health? Frontiers in cellular and infection microbiology 5: 35 .

40. Lehmann-Kalata AP, Surdacka A, Ciężka-Hsiao E, Swora-Cwynar E, Grzymisławski M, et al. (2015) Clinical parameters of oral cavity, physical and microbiological properties of saliva in patients with obesity. Dental and Medical Problems 52(4): 415-423.

41. Li X, Kolltveit KM, Tronstad L, Olsen I (2000) Systemic diseases caused by oral infection. Clinical microbiology reviews 13(4): 547-558.

42. Lim Y, Fukuma N, Totsika M, Kenny L, Morrison M, et al. (2018) The performance of an oral microbiome biomarker panel in predicting oral cavity and oropharyngeal cancers. Frontiers in cellular and infection microbiology 8: 267.

43. Madianos PN, Bobetsis YA, Offenbacher S (2013) Adverse pregnancy outcomes (APOs) and periodontal disease: pathogenic mechanisms. Journal of clinical periodontology 40: S170-S180.

44. Mager DL, Haffajee AD, Devlin PM, Norris CM, Posner MR, et al. (2005) The salivary microbiota as a diagnostic indicator of oral cancer: a descriptive, non-randomized study of cancer-free and oral squamous cell carcinoma subjects. Journal of translational medicine 3(1): 27.

45. Michaud DS, Izard J (2014) Microbiota, oral microbiome, and pancreatic cancer. Cancer journal (Sudbury, Mass.) 20(3): 203-206.

46. Morre SA, Stooker W, Lagrand WK, Van den Brule AJC, Niessen HWM, et al. (2000) Microorganisms in the aetiology of atherosclerosis. Journal of clinical pathology 53(9): 647-654. 
47. Naidoo T, Konkol K, Mckune AJ, Biccard B, Dubose K, et al. (2012) Elevated salivary C-reactive protein predicted by low cardio-respiratory fitness and being overweight in African children. Cardiovascular Journal of Africa 23(9): 501-506.

48. Orr ME, Reveles KR, Yeh CK, Young EH, Han X, et al. (2019) Can Oral Health and Oral-derived Biospecimens Predict Progression of Dementia? Oral diseases 26(2): 249-258.

49. Panariello BHD, de Araújo Costa CAG, Pavarina AC, Santiago SL, Duarte S, et al. (2017) Advances and challenges in oral biofilm control. Current Oral Health Reports 4(1): 29-33.

50. Paster BJ, Olsen I, Aas JA, Dewhirst FE (2006) The breadth of bacterial diversity in the human periodontal pocket and other oral sites. Periodontology 2000 42(1): 80-87.

51. Peters BA, Wu J, Pei Z, Yang L, Purdue MP, et al. (2017) Oral microbiome composition reflects prospective risk for esophageal cancers. Cancer research 77(23): 6777-6787.

52. Piconi S, Trabattoni D, Luraghi C, Perilli E, Borelli M, et al. (2009) Treatment of periodontal disease results in improvements in endothelial dysfunction and reduction of the carotid intima-media thickness. The FASEB Journal 23(4): 1196-1204.

53. Poole S, Singhrao SK, Kesavalu L, Curtis MA, Crean S, et al. (2013) Determining the presence of periodontopathic virulence factors in short-term postmortem Alzheimer's disease brain tissue. Journal of Alzheimer's Disease 36(4): 665-677.

54. Pradeep AR, Priyanka N, Prasad MVR, Kalra N, Kumari M, et al. (2012) Association of progranulin and high sensitivity CRP concentrations in gingival crevicular fluid and serum in chronic periodontitis subjects with and without obesity. Disease markers 33(4): 207-213.

55. Pussinen PJ, Tuomisto K, Jousilahti P, Havulinna AS, Sundvall J, et al (2007) Endotoxemia, immune response to periodontal pathogens, and systemic inflammation associate with incident cardiovascular disease events. Arteriosclerosis, thrombosis, and vascular biology 27(6): 14331439.

56. Range H, Poitou C, Boillot A, Ciangura C, Katsahian S, et al. (2013) Orosomucoid, a new biomarker in the association between obesity and periodontitis. PloS one 8(3): e57645.

57. Recker EN, Brogden KA, Avila-Ortiz G, Fischer CL, Pagan-Rivera K, et al. (2015) Novel biomarkers of periodontitis and/or obesity in saliva-An exploratory analysis. Archives of oral biology 60(10): 1503-1509.

58. Said HS, Suda W, Nakagome S, Chinen H, Oshima K, et al. (2014) Dysbiosis of salivary microbiota in inflammatory bowel disease and its association with oral immunological biomarkers. DNA research 21(1): 15-25.

59. Sampaio-Maia B, Caldas IM, Pereira ML, Pérez-Mongiovi D, Araujo R, et al. (2016) The oral microbiome in health and its implication in oral and systemic diseases. In Advances in applied microbiology Academic Press 97: 171-210.

ISSN: 2574-1241

DOI: 10.26717/BJSTR.2020.30.004993

Subhash Padhye. Biomed J Sci \& Tech Res

(C) (P) This work is licensed under Creative

Submission Link: https://biomedres.us/submit-manuscript.php
60. Shoemark DK, Allen SJ (2015) The microbiome and disease: reviewing the links between the oral microbiome, aging, and Alzheimer's disease. Journal of Alzheimer's Disease 43(3): 725-738.

61. Singh H, Torralba, MG, Moncera KJ, DiLello L, Petrini J, et al. (2019) Gastro-intestinal and oral microbiome signatures associated with healthy aging. GeroScience 41(6): 907-921.

62. Sohail MU, Elrayess MA, Al Thani AA, Al-Asmakh M, Yassine HM, et al. (2019) Profiling the Oral Microbiome and Plasma Biochemistry of Obese Hyperglycemic Subjects in Qatar. Microorganisms 7(12): 645.

63. Tam J, Hoffmann T, Fischer S, Bornstein S, Graessler J, et al. (2018) Obesity alters composition and diversity of the oral microbiota in patients with type 2 diabetes mellitus independently of glycemic control. PloS one 13(10): e0204724.

64. Verma D, Garg PK, Dubey AK (2018) Insights into the human oral microbiome. Archives of microbiology 200(4): 525-540.

65. Wolf A, Moissl-Eichinger C, Perras A, Koskinen K, Tomazic PV, et al. (2017) The salivary microbiome as an indicator of carcinogenesis in patients with oropharyngeal squamous cell carcinoma: A pilot study. Scientific reports 7(1): 1-10.

66. Xu Y, Zhang M, Zhang J, Sun Z, Ran L, et al. (2019) Differential Intestinal and Oral Microbiota Features Associated with Gestational Diabetes and Maternal Inflammation. American Journal of Physiology-Endocrinology and Metabolism 319(2): E247-E253.

67. Yamashita Y, Takeshita T (2017) The oral microbiome and human health. Journal of oral science, 59(2): 201-206.

68. Yan X, Yang M, Liu J, Gao R, Hu J, et al. (2015) Discovery and validation of potential bacterial biomarkers for lung cancer. American journal of cancer research 5(10): 3111-3122.

69. Zaremba M, Górska R, Suwalski P, Kowalski J (2007) Evaluation of the incidence of periodontitis-associated bacteria in the atherosclerotic plaque of coronary blood vessels. Journal of periodontology 78(2): 322327.

70. Zaura E, Nicu E A, Krom BP, Keijser BJ (2014) Acquiring and maintaining a normal oral microbiome: current perspective. Frontiers in cellular and infection microbiology 4: 85.

71.Zeigler CC, Persson GR, Wondimu B, Marcus C, Sobko T, et al. (2012) Microbiota in the oral subgingival biofilm is associated with obesity in adolescence. Obesity 20(1): 157-164

72. Zhou Y, Gao H, Mihindukulasuriya KA, La Rosa PS, Wylie KM, et al. (2013) Biogeography of the ecosystems of the healthy human body. Genome biology 14(1): R1.

73. (2018)All Human Oral Microbial Taxa.

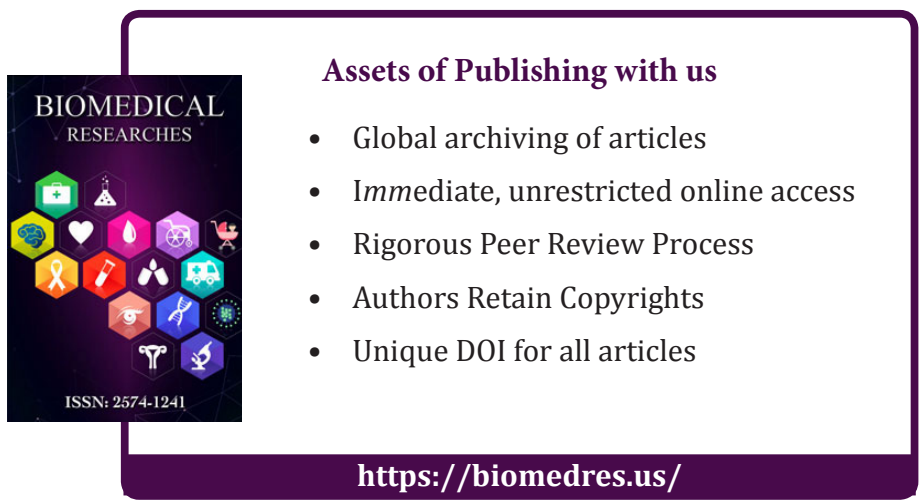

